\title{
Association between serum tumor markers and metabolic tumor volume or total lesion glycolysis in patients with recurrent small cell lung cancer
}

\author{
PENGYUE SHI ${ }^{*}$, XUE MENG* ${ }^{*}$ MENGMENG NI, XINDONG SUN, LIGANG XING and JINMING YU
}

Department of Radiation Oncology, Shandong Cancer Hospital, Shandong University, Jinan, Shandong 250117, P.R. China

Received October 19, 2014; Accepted July 16, 2015

DOI: $10.3892 / \mathrm{ol} .2015 .3673$

\begin{abstract}
The aim of the present study was to investigate the association between serum tumor markers and the metabolic tumor volume (MTV) or total lesion glycolysis (TLG), as determined by fluorine-18 fluorodeoxyglucose $\left({ }^{18} \mathrm{~F}-\mathrm{FDG}\right)$ positron emission tomography-computed tomography (PET/CT) in patients with recurrent small cell lung cancer (SCLC). Data from 21 patients with recurrent SCLC were collected. The levels of neuron-specific enolase (NSE), carcinoembryonic antigen (CEA) and cytokeratin 19 fragment 21-1 were measured at the time of the ${ }^{18} \mathrm{~F}-\mathrm{FDG} \mathrm{PET} / \mathrm{CT}$ examination. The MTV and TLG of all lesions were calculated. Pearson correlation analyses were used to estimate the correlations between NSE level and PET findings. Pearson correlation analyses showed that NSE was the only tumor marker to have a strong correlation with MTV or TLG $(r=0.787, \mathrm{P}<0.001$; $\mathrm{r}=0.866, \mathrm{P}<0.001$, respectively). In patients with a normal NSE level, no correlation was found between NSE and MTV or TLG ( $\mathrm{r}=0.018, \mathrm{P}=0.958 ; \mathrm{r}=-0.003, \mathrm{P}=0.92$, respectively), but a significant correlation was found in patients with an abnormal NSE level ( $\mathrm{r}=0.789, \mathrm{P}<0.01 ; \mathrm{r}=0.872, \mathrm{P}=0.01$, respectively). Therefore, TLG and MTV may serve as sensitive markers of tumor burden in patients with recurrent SCLC, with TLG showing greater sensitivity. In patients with an abnormal NSE level, a higher NSE level indicates greater MTV and TLG.
\end{abstract}

\section{Introduction}

Lung cancer is one of the most common cancer types in the world. Small cell lung cancer (SCLC) represents 15-20\% of all

Correspondence to: Mr. Jinming Yu, Department of Radiation Oncology, Shandong Cancer Hospital, Shandong University, 440 Jiyan Road, Jinan, Shandong 250117, P.R. China

E-mail: sdszlyyyjm@126.com

*Contributed equally

Key words: small cell lung cancer, neuron-specific enolase, metabolic tumor volume, total lesion glycolysis, positron emission tomography lung cancers (1). This disease is characterized by rapid tumor growth and early metastatic spread (2), which lead to a high rate of relapse and a poor prognosis. Since the 1980s, the standard chemotherapy for SCLC has been a cisplatin-etoposide combination. Practically all affected patients are treated with chemotherapy, either alone or in combination with a local therapy, such as radiation therapy. Although SCLC is one of the most chemo-sensitive solid tumors (3), recurrences occur in the majority of patients, particularly in the first year (4).

As neuroendocrine differentiation is considered to be an important feature of SCLC, neuron-specific enolase (NSE) has been utilized as a marker for its diagnosis and therapeutic monitoring. Moreover, it has been found that the levels of this marker have prognostic value. A previous study found that the percentage change in NSE level correlated with the percent decrease in the sum of the tumor diameters in patients with SCLC (5). Therefore, the serum NSE level may be associated with the tumor burden in SCLC patients.

Fluorine-18 fluorodeoxyglucose $\left({ }^{18} \mathrm{~F}-\mathrm{FDG}\right) \mathrm{PET} / \mathrm{CT}$ (PET/CT) is a hybrid imaging modality that is able to provide functional and anatomical information. Successful FDG PET scanning has been performed in a wide variety of cancers and been identified as a sensitive imaging tool in a number of studies, based on its usefulness in the detection of recurrence, accurate staging and the impact on the management of SCLC (6). In patients with recurrent colorectal cancer, a correlation has been found between serum carcinoembryonic antigen (CEA) and metabolic tumor volume (MTV), as determined by FDG PET (7), which indicates that tumor volume determined by FDG PET can be utilized as an effective marker of the tumor burden in post-operative colorectal carcinoma patients. The present study was undertaken to investigate the correlation between tumor MTV or total lesion glycolysis (TLG), as determined by ${ }^{18} \mathrm{~F}-\mathrm{FDG}$ PET/CT, and the serum level of tumor markers in recurrent SCLC patients. This may serve as a basic study for tumor markers and FDG PET use in recurrent SCLC.

\section{Patients and methods}

Between October 2009 and December 2011, a search was performed for all patients who had been diagnosed with recurrent SCLC in the PET center database of the Shandong Cancer Hospital (Shandong University, Jinan, Shandong, China). 
The patient selection criteria were for recurrent patients who had been previously treated by standard chemotherapy with a response evaluation showing complete remission. Serum CEA, NSE and cytokeratin 19 fragment 21-1 (CYFRA 21-1) levels were evaluated at the time of the ${ }^{18} \mathrm{~F}-\mathrm{FDG}$ PET/CT examination. A total of 21 patients were enrolled in the present study (Table I). Of these, 17 patients had lactate dehydrogenase ( $\mathrm{LDH}$ ) data. The mean age of the patients was 62.2 years (range, 37-79 years). The patients were staged according to a two-stage system that divided SCLC into limited disease (LD) and extensive disease (ED). LD was defined as disease confined to the ipsilateral hemithorax that can be safely included in a tolerable radiation field, and all remaining cases were considered to be ED (8). Of the 21 patients, 14 were staged as LD and 7 as ED. Based on the lesion site, FDG PET revealed that 18 patients presented with recurrent lung lesions, 10 patients with lymph node lesions, 3 patients with bone lesions and 1 patient with an adrenal gland lesion (Table II). All recurrent or metastatic lesions were verified according to histological analysis. Ethical approval was obtained from the Ethics Committee of Shandong University and the study was performed according to the Declaration of Helsinki (2013) (9).

FDG-PET imaging protocol. Whole-body PET scans were performed using a Xeleris workstation (GE Healthcare Life Sciences, Shanghai, China). All patients fasted for at least $6 \mathrm{~h}$ prior to the intravenous administration of FDG. Image acquisitions for torso scanning were started at $\sim 1 \mathrm{~h}$ after the injection of $7.4 \mathrm{MBq}$ FDG per kilogram of body weight, and regional emission images were obtained for $30 \mathrm{~min}$ in the two-dimensional mode. Transmission scanning with three germanium-68 ring sources was performed for 2 min per bed in whole-body transmission and for $20 \mathrm{~min}$ in regional transmission to correct attenuation. Images were visually interpreted by consensus between two experienced nuclear physicians. Standardized uptake values (SUV) were calculated from the amount of FDG injected, the body weight and the target tissue uptake in regional attenuation corrected images.

MTV and TLG of tumors. For the various methods for metabolic volume measurement, an SUV cut-off of 2.5 was used. This meant that the PET area was delineated by a circle encompassing regions equal or greater than a SUV of 2.5. The MTV of each slice was determined by multiplying the area within the thresholded margin by the CT interval. The final MTV was calculated by adding the MTVs of each slice. Maximum and mean SUVs within the MTV were calculated automatically, and TLG was calculated by multiplying the

$$
\begin{aligned}
& M T V=\sum_{i=1}^{n} S_{i} \times d \\
& T L G=\sum_{i=1}^{n} M T V_{i} \times S U \text { Vmean }_{i}
\end{aligned}
$$

MTV by the mean SUV. The following formulae were used to calculate MTV and TLG:

$\mathrm{S}_{\mathrm{i}}$ represents the area with abnormal metabolism of each slice, $d$ represents the interval of the CT scan, $\mathrm{MTV}_{\mathrm{i}}$ represents the MTV of each slice; $\mathrm{SUVmean}_{\mathrm{i}}$ represents the mean
Table I. Basic information for 21 patients.

Characteristic

Value

$\begin{array}{lc}\text { Age, years } & \\ \text { Range } & 37-79 \\ \text { Mean } & 62.29 \\ <60, \mathrm{n}(\%) & 10(47.6) \\ >60, \mathrm{n}(\%) & 11(52.4) \\ \text { Gender, } \mathrm{n}(\%) & \\ \text { Male } & 17(81.0) \\ \text { Female } & 4(19.0) \\ \text { Staging, }(\%) & \\ \text { LD } & 14(66.7) \\ \text { ED } & 7(33.3) \\ \text { NSE level, }(\%) & \\ >17 \text { ng/ml } & 11(52.4) \\ <17 \text { ng/ml } & 10(47.6) \\ \text { Number of lesions, } \mathrm{n}(\%) & \\ \text { One } & 12(57.1) \\ \text { Two } & 6(28.6) \\ \text { Three } & 3(14.3) \\ \text { Lesion sites, } \mathrm{n}(\%) & \\ \text { Lungs } & 18(85.7) \\ \text { Lymph node } & 10(47.6) \\ \text { Adrenal } & 1(4.8) \\ \text { Bone } & 3(14.3) \\ \end{array}$

LD, limited disease; ED, extensive disease; NSE, neuron-specific enolase.

SUV of each slice and $\mathrm{n}$ represents the number of slices with abnormal metabolism.

Statistical analysis. All data were analyzed using Statistical Package for the Social Sciences (SPSS) 17.0 software (SPSS, Inc., Chicago, IL, USA). Differences between groups were analyzed using an independent two-sample t-test. The Pearson rank correlation analysis was used to evaluate the correlation between two groups. $\mathrm{P}<0.05$ was used to indicate a statistically significant difference.

\section{Result}

In 21 patients, the mean serum NSE level was $35.38 \pm 61.03 \mathrm{ng} / \mathrm{ml}$ (range, 8.26-293.80 ng/ml); 11 patients exhibited normal NSE levels $(<17 \mathrm{ng} / \mathrm{ml})$, while the other 10 exhibited abnormal levels. The mean serum CEA level was $22.58 \pm 86.14 \mathrm{ng} / \mathrm{ml}$ (range, $0.67-398.10 \mathrm{ng} / \mathrm{ml}$ ) and the mean CYFRA 21-1 level was $2.97 \pm 1.91 \mathrm{ng} / \mathrm{ml}$ (range, $1.11-8.83 \mathrm{ng} / \mathrm{ml}$ ). The mean MTV was $45.837 \pm 58.676 \mathrm{~cm}^{3}$ (range, $0.450-213.786 \mathrm{~cm}^{3}$ ), while the mean TLG was $217.417 \pm 320.788 \mathrm{~cm}^{3}$ (range, $1.363-1283.235 \mathrm{~cm}^{3}$ ).

Pearson correlation analyses showed a strong correlation between the NSE level and MTV or TLG ( $r=0.787, \mathrm{P}<0.001$; $\mathrm{r}=0.866, \mathrm{P}<0.001$, respectively; Fig. 1). The $t$-test showed that the MTV and TLG of the patients with abnormal NSE levels were significantly higher than those of patients with normal NSE 
A

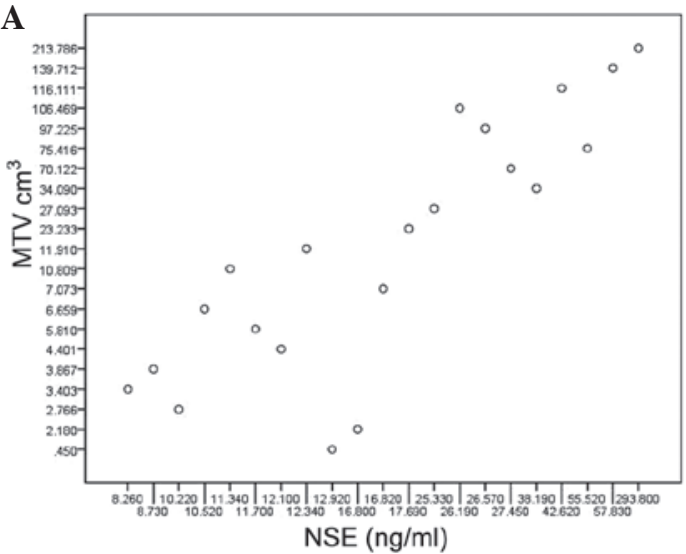

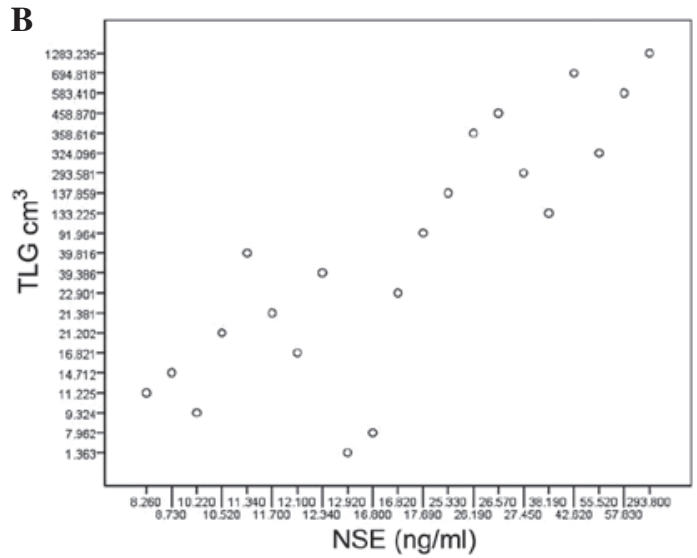

Figure 1. Pearson correlation analyses indicating a strong correlation between neuron-specific enolase (NSE) level and (A) metabolic tumor volume (MTV) or (B) total lesion glycolysis (TLG) $(r=0.787, \mathrm{P}<0.001 ; \mathrm{r}=0.866, \mathrm{P}<0.001$, respectively).
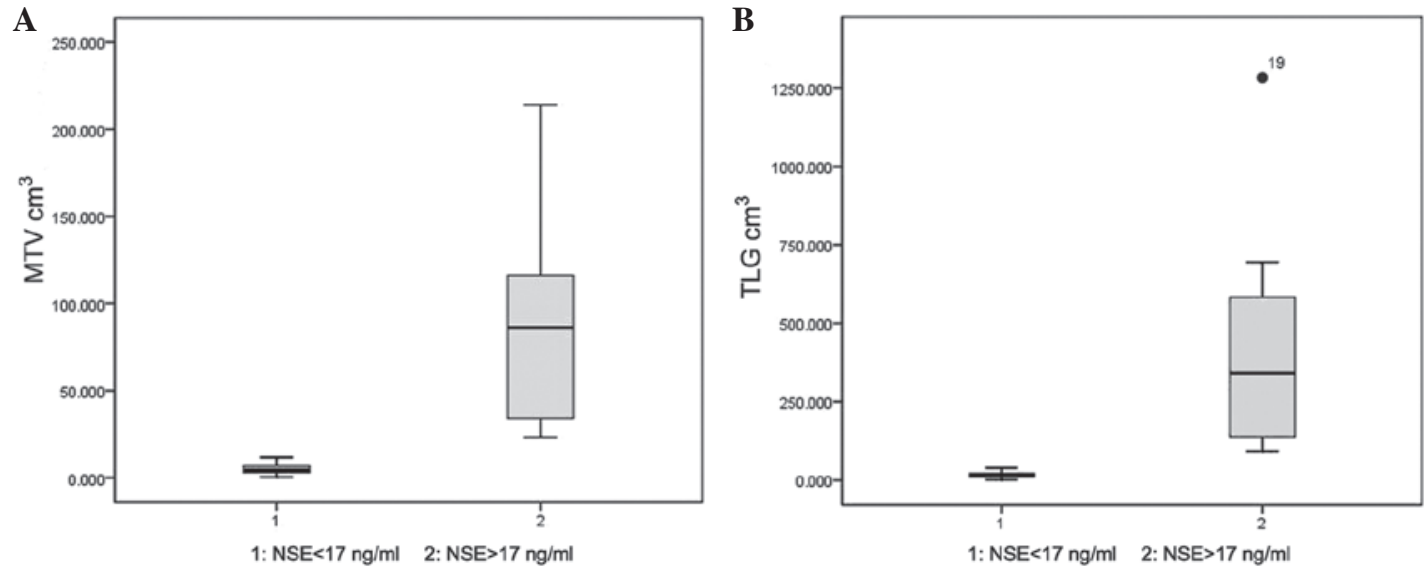

Figure 2. Results of the t-test indicating that the (A) metabolic tumor volume (MTV) and (B) total lesion glycolysis (TLG) of patients with abnormal neuron-specific enolase (NSE) levels were significantly higher than those of patients with normal NSE levels ( $\mathrm{P}=0.001$ and $\mathrm{P}=0.002$, respectively).
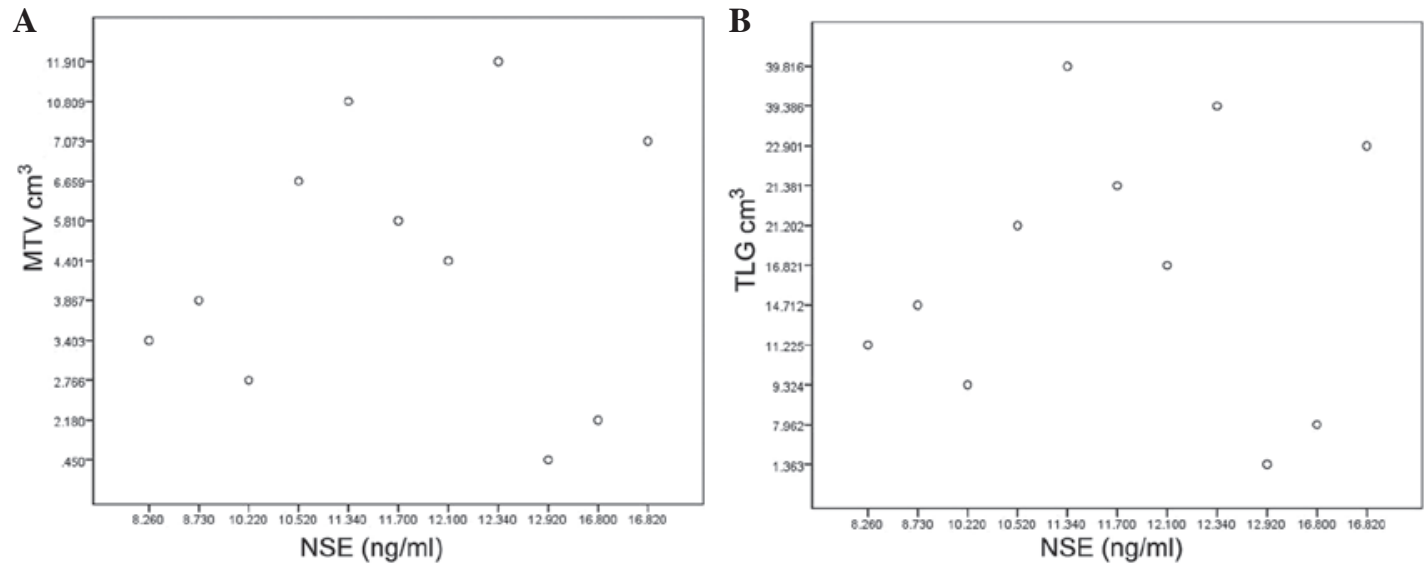

Figure 3. Pearson correlation analyses indicating that in patients with normal neuron-specific enolase (NSE) levels, no correlation was present between NSE and (A) metabolic tumor volume (MTV) or (B) total lesion glycolysis (TLG) $(r=0.018, \mathrm{P}=0.958 ; \mathrm{r}=-0.003, \mathrm{P}=0.92$, respectively).

levels ( $\mathrm{P}=0.001$ and $\mathrm{P}=0.002$, respectively; Fig. 2). In the patients with normal NSE levels, no correlation was found between NSE and MTV or TLG $(r=0.018, \mathrm{P}=0.958 ; \mathrm{r}=-0.003, \mathrm{P}=0.92$, respectively; Fig. 3), but the correlation was significant in patients with abnormal NSE levels $(\mathrm{r}=0.789, \mathrm{P}<0.01 ; \mathrm{r}=0.872, \mathrm{P}=0.01$, respectively; Fig. 4). In the 17 patients with evaluated LDH levels, the
LDH level was also correlated with MTV and TLG ( $\mathrm{r}=0.656$, $\mathrm{P}=0.004 ; \mathrm{r}=0.697, \mathrm{P}=0.002$, respectively). However, no such correlation was found between CEA level and MTV or TLG ( $r=-0.039, P=0.866 ; r=-0.054, P=0.817$, respectively). Similarly, no correlation was found between CYFRA 21-1 level and MTV or TLG ( $r=0.263, \mathrm{P}=0.250 ; \mathrm{r}=0.245, \mathrm{P}=0.284$, respectively). 
Table II. Data on tumor markers, MTV and TLG for 21 patients.

\begin{tabular}{|c|c|c|c|c|c|c|c|}
\hline Patient no. & NSE, ng/ml & $\mathrm{LDH}, \mathrm{U} / 1$ & CEA, ng/ml & CYFRA 21-1, ng/ml & Lesion sites & $\mathrm{MTV}, \mathrm{cm}^{3}$ & TLG, $\mathrm{cm}^{3}$ \\
\hline 1 & 8.73 & 176 & 1.160 & 1.67 & Lung & 3.867 & 14.712 \\
\hline 2 & 16.82 & 191 & 1.260 & 2.07 & Lung & 7.073 & 22.901 \\
\hline 3 & 8.26 & 140 & 1.600 & 1.94 & Lung & 3.403 & 11.225 \\
\hline 4 & 10.52 & 192 & 17.490 & 1.82 & Lung & 6.659 & 21.202 \\
\hline 5 & 12.34 & 185 & 0.676 & 1.55 & Lung & 11.910 & 39.386 \\
\hline 6 & 12.10 & 142 & 1.360 & 1.14 & Lymph node & 4.401 & 16.821 \\
\hline 7 & 38.19 & 232 & 398.100 & 5.09 & Lung, bone & 34.090 & 133.225 \\
\hline 8 & 12.92 & 165 & 2.230 & 1.43 & Lung & 0.450 & 1.363 \\
\hline 9 & 11.70 & 141 & 0.912 & 1.47 & Lymph node & 5.810 & 21.381 \\
\hline 10 & 27.45 & 225 & 1.670 & 4.80 & Lung, lymph node & 70.122 & 293.581 \\
\hline 11 & 26.57 & 129 & 2.730 & 2.43 & Lung, lymph node & 97.225 & 458.870 \\
\hline 12 & 57.83 & 158 & 1.040 & 3.35 & Lung, lymph node & 139.712 & 583.410 \\
\hline 13 & 55.52 & 384 & 9.020 & 8.83 & Lung, lymph node, bone & 75.416 & 324.096 \\
\hline 14 & 42.62 & 241 & 9.990 & 4.42 & Lung & 116.111 & 694.818 \\
\hline 15 & 11.34 & 141 & 3.350 & 5.56 & Lung & 10.809 & 39.816 \\
\hline 16 & 26.19 & NA & 7.190 & 1.27 & Lung, lymph node & 106.469 & 358.616 \\
\hline 17 & 10.22 & NA & 2.610 & 3.54 & Lung & 2.766 & 9.324 \\
\hline 18 & 17.69 & NA & 3.860 & 1.11 & Lung, lymph node & 23.233 & 91.964 \\
\hline 19 & 293.80 & 455 & 3.390 & 3.09 & Lymph node, adrenal & 213.786 & 1283.235 \\
\hline 20 & 25.33 & 169 & 1.350 & 3.35 & Lung, lymph node, bone & 27.093 & 137.859 \\
\hline 21 & 16.80 & NA & 3.230 & 2.55 & Lung & 2.180 & 7.962 \\
\hline
\end{tabular}

NA, not available; MTV, metabolic tumor volume; TLG, total lesion glycolysis; NSE, neuron-specific enolase; LDH, lactate dehydrogenase; CEA, carcinoembryonic antigen; CYFRA 21-1, cytokeratin 19 fragment 21-1.

A

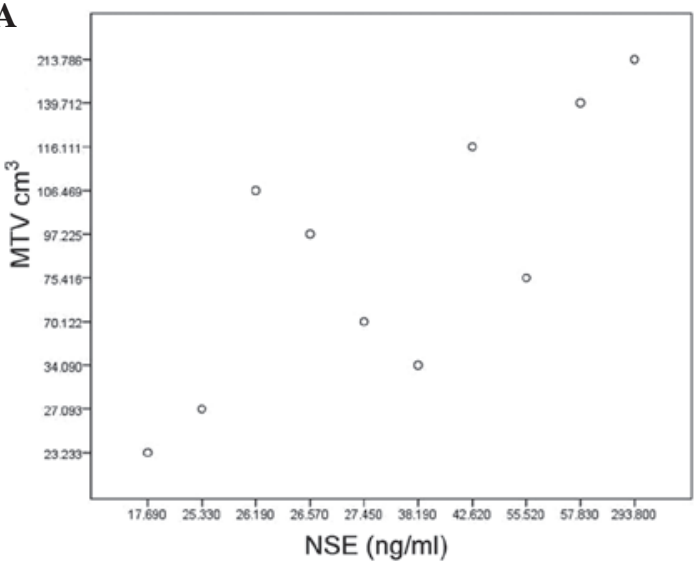

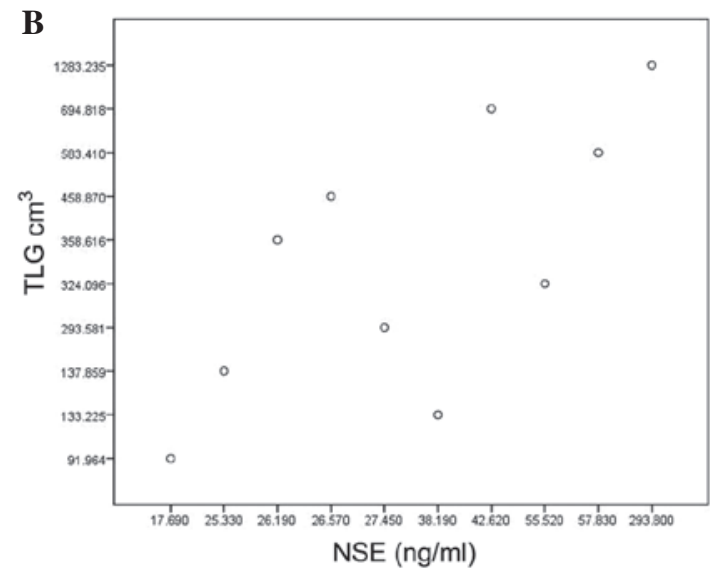

Figure 4. Pearson correlation analyses indicating that in patients with abnormal neuron-specific enolase (NSE) levels, a significant correlation was present between NSE and (A) metabolic tumor volume (MTV) or (B) total lesion glycolysis (TLG) $(\mathrm{r}=0.789, \mathrm{P}<0.01 ; \mathrm{r}=0.872, \mathrm{P}=0.01, \mathrm{respectively)}$.

\section{Discussion}

In the present retrospective study, a strong correlation was found between serum NSE level and MTV or TLG, as determined by FDG PET in recurrent SCLC patients, while serum CEA and CYFRA 21-1 levels showed no correlation with MTV or TLG.

Knowledge of the tumor burden is useful in cancer patients, as total tumor burden is often associated with tumor response to therapy and has significant effect on the prognosis $(10,11)$. SCLC is a highly aggressive tumor with a high relapse rate; therefore, finding a method to accurately evaluate the tumor burden of recurrent SCLC patients is a high priority.

CEA, NSE and CYFRA 21-1 are used widely in lung cancer patients (12). Among these three tumor markers, NSE is considered to be the most sensitive to SCLC; this has been confirmed by numerous studies since Carney et al (13) first proposed NSE as a tumor marker for SCLC. Serum levels of NSE are elevated in $40-45 \%$ of patients with LD and in $85-98 \%$ of patients with ED (14). Bonner et al (15) reported that pretreatment serum NSE levels may reflect the tumor burden and predict survival, and that higher NSE level indicate a worse prognosis, suggesting 
that NSE level can be used to assess the prognosis of SCLC patients. Consistent with the aforementioned findings, among the three lung cancer-related tumor markers studied, the present study also determined that NSE was the only marker with a correlation with MTV and TLG, as determined by FDG PET in recurrent SCLC patients. However, the clinical application of NSE has certain limitations, as a normal NSE level does not exclude the existence of a tumor. Therefore, the use of the NSE level as an effective marker for tumor burden of recurrent SCLC patients is not recognized. A previous study demonstrated that $\mathrm{LDH}$ has prognostic value (16) and may serve as an effective marker in SCLC. The present study also found that LDH exhibited a correlation with MTV and TLG in the 17 patients who had LDH data.

Currently, in the clinic, the evaluation of the tumor burden usually depends on the maximum diameter of the tumor and the tumor volume, as measured by CT scans. However, CT has certain shortcomings, such as the inability to distinguish between tumor and necrotic tissues. Furthermore, small tumors with diameters similar or smaller than the slice thickness cannot be visualized on CT imaging. Above all, CT scans usually poorly represent the tumor burden (17).

These disadvantages can, however, be overcome by the use of FDG PET. PET imaging has the advantage of being able to detect and distinguish between slight metabolic changes prior to structural changes in tissues and organs occurring in vivo. FDG PET-CT imaging is considered to fuse the functional image of PET and the anatomical image of CT. Certain studies $(18,19)$ found that on radiation therapy treatment planning, the sketch of the tumor volume under PET-CT was changed in $>50 \%$ of patients compare with CT-based treatment planning. MTV and TLG are critical and sensitive indices with regard to tumor metabolism. The prognostic significance of metabolic parameters measured by FDG PET-CT, such as MTV, TLG and maximum SUV, has been reported in a variety of cancers, including lung cancer, esophageal cancer and lymphoma (20-23). Certainly, in SCLC, metabolic parameters are also considered to be of prognostic value (24). Furthermore, the retrospective study conducted by Xie et al found that TLG, which combined MTV and SUVmean, showed greater prognostic value in nasopharyngeal carcinoma compared with MTV (25). The present study showed that TLG exhibited a greater correlation coefficient than MTV with serum NSE level, which, to a certain extent, supports the theory that TLG has a greater prognostic value than MTV.

In the present study, the serum NSE level exhibited no correlation with MTV or TLG in the patients with normal NSE levels, while the correlation was significant in the patients with abnormal NSE levels. It is known that patients with normal NSE levels usually have relatively small tumors. In such tumor tissue, the peripheral inflammatory cells and granulation tissue, particularly activated macrophages and young granulation tissue, exhibit significant FDG uptake (26). Therefore, inflammatory cells and granulation tissue may have greater influence on MTV and TLG in patients with small tumors compared with large tumors. This may explain why serum NSE levels showed no correlation with MTV or TLG in the patients with a normal NSE level.

However, the present study also had certain limitations, such as the retrospective nature of the study design, the artificially sketched tumor margin, leading to inevitable deviation of the MTV and TLG calculations, and the relatively small number of patients in the cohort. Despite these shortcomings, the present study has value, as it provides an effective method to accurately evaluate the tumor burden in patients with recurrent SCLC.

In conclusion, the present study found a significant correlation between NSE and MTV or TLG. MTV and TLG, as determined by FDG PET, can be used effectively to assess the tumor burden in patients with recurrent SCLC, with TLG being more sensitive than MTV. When diagnosed as recurrent SCLC, a higher NSE level suggested a heavier tumor burden in patients with an abnormal NSE level. Controlled prospective studies in a larger patient cohort is required to validate these findings. The study could provide the theoretical foundation for the further application of tumor markers and FDG PET in patients with recurrent SCLC.

\section{Acknowledgements}

The authors would like to thank Dr Zhu Wanqi and Dr Ma Li for providing assistance with this study. The study was presented in part as a poster presentation at the 55th Annual Meeting of the American Society for Radiation Oncology, Oct 22-25, 2013, Atlanta, GA, USA.

\section{References}

1. Parkin DM, Bray F, Ferlay $\mathrm{J}$ and Pisani P: Global cancer statistics, 2002. CA Cancer J Clin 55: 74-108, 2005.

2. van Meerbeeck JP, Fennell DA and De Ruysscher DK: Small-cell lung cancer. Lancet 378: 1741-1755, 2011.

3. Johnson BE and Jänne PA: Basic treatment considerations using chemotherapy for patients with small cell lung cancer. Hematol Oncol Clin North Am 18: 309-322, 2004.

4. Simon M, Argiris A and Murren JR: Progress in the therapy of small cell lung cancer. Crit Rev Oncol Hematol 49: 119-133, 2004.

5. Ono A, Naito T, Ito I, Watanabe R, Shukuya T, Kenmotsu H, Tsuya A, Nakamura Y, Murakami H, Kaira K, et al: Correlations between serial pro-gastrin-releasing peptide and neuron-specific enolase levels and the radiological response to treatment and survival of patients with small-cell lung cancer. Lung cancer 76: 439-444, 2012

6. Fischer BM, Mortensen J, Langer SW, Loft A, Berthelsen AK, Petersen BI, Daugaard G, Lassen U and Hansen HH: A prospective study of PET/CT in initial staging of small-cell lung cancer: Comparison with $\mathrm{CT}$, bone scintigraphy and bone marrow analysis. Ann Oncol 18: 338-345, 2007.

7. Choi MY, Lee KM, Chung JK, Lee DS, Jeong JM, Park JG, Kim JH and Lee MC: Correlation between serum CEA level and metabolic volume as determined by FDG PET in postoperative patients with recurrent colorectal cancer. Ann Nucl Med 19: 123-129, 2005.

8. Simon GR and Wagner H; American College of Chest Physicians: Small cell lung cancer. Chest 123 (Suppl 1): 259S-271S, 2003.

9. World Medical Association: World Medical Association Declaration of Helsinki: Ethical principles for medical research involving human subjects. JAMA 310: 2191-2194, 2013.

10. Johnson CR, Khandelwal SR, Schmidt-Ullrich RK, Ravalese J III and Wazer DE: The influence of quantitative tumor volume measurements on local control in advanced head and neck cancer using concomitant boost accelerated superfractionated irradiation. Int J Radiat Oncol Biol Phys 32: 635-641, 1995.

11. Bradley JD, Ieumwananonthachai N, Purdy JA, Wasserman TH, Lockett MA, Graham MV and Perez CA: Gross tumor volume, critical prognostic factor in patients treated with three-dimensional conformal radiation therapy for non-small-cell lung carcinoma. Int J Radiat Oncol Biol Phys 52: 49-57, 2002.

12. Harmsma M, Schutte B and Ramaekers FC: Serum markers in small cell lung cancer: Opportunities for improvement. Biochim Biophys Acta 1836: 255-272, 2013. 
13. Carney DN, Marangos PJ, Ihde DC, Bunn PA Jr, Cohen MH, Minna JD and Gazdar AF: Serum neuron-specific enolase: A marker for disease extent and response to therapy of small-cell lung cancer. Lancet 1: 583-585, 1982.

14. Jorgensen LG: Neuron specific enolase in small cell lung cancer. Clinical and biochemical evaluation. Dan Med Bull 46: 1-12, 1999.

15. Bonner JA, Sloan JA, Rowland KM Jr, Klee GG, Kugler JW, Mailliard JA, Wiesenfeld M, Krook JE, Maksymiuk AW, Shaw EG, et al: Significance of neuron-specific enolase levels before and during therapy for small cell lung cancer. Clin Cancer Res 6: 597-601, 2000

16. Yip D and Harper PG: Predictive and prognostic factors in small cell lung cancer: Current status. Lung cancer 28: 173-185, 2000.

17. Jennings SG1, Winer-Muram HT, Tarver RD and Farber MO: Lung tumor growth: Assessment with CT - comparison of diameter and cross-sectional area with volume measurements. Radiology 231: 866-871, 2004

18. Ciernik IF, Dizendorf E, Baumert BG, Reiner B, Burger C, Davis JB, Lütolf UM, Steinert HC and Von Schulthess GK: Radiation treatment planning with an integrated positron emission and computer tomography (PET/CT): A feasibility study. Int J Radiat Oncol Biol Phys 57: 853-863, 2003.

19. Bradley J, Thorstad WL, Mutic S, Miller TR, Dehdashti F, Siegel BA, Bosch W and Bertrand RJ: Impact of FDG-PET on radiation therapy volume delineation in non-small-cell lung cancer. Int J Radiat Oncol Biol Phys 59: 78-86, 2004.

20. Liao S, Penney BC, Wroblewski K, Zhang H, Simon CA, Kampalath R, Shih MC, Shimada N, Chen S, Salgia R, et al: Prognostic value of metabolic tumor burden on 18F-FDG PET in nonsurgical patients with non-small cell lung cancer. Eur J Nucl Med Mol Imaging 39: 27-38, 2012.
21. Vu CC, Matthews R, Kim B, Franceschi D, Bilfinger TV and Moore WH: Prognostic value of metabolic tumor volume and total lesion glycolysis from 18F-FDG PET/CT in patients undergoing stereotactic body radiation therapy for stage I non-small-cell lung cancer. Nucl Med Commun 34: 959-963, 2013.

22. Roedl JB, Halpern EF, Colen RR, Sahani DV, Fischman AJ and Blake MA: Metabolic tumor width parameters as determined on PET/CT predict disease-free survival and treatment response in squamous cell carcinoma of the esophagus. Mol Imaging Biol 11: 54-60, 2009.

23. Oh MY, Chung JS, Song MK, Shin HJ, Lee HS, Lee SM, Lee GW and Lee SE: Prognostic value of Waldeyer's ring involvement of diffuse large B-cell lymphoma treated with R-CHOP. Int J Hematol 97: 397-402, 2013.

24. Zhu D, Ma T, Niu Z, Zheng J, Han A, Zhao S and $\mathrm{Yu}$ J: Prognostic significance of metabolic parameters measured by (18)F-fluorodeoxyglucose positron emission tomography/computed tomography in patients with small cell lung cancer. Lung cancer 73: 332-337, 2011.

25. Xie P, Yue JB, Zhao HX, Sun XD, Kong L, Fu Z and Yu JM Prognostic value of 18 F-FDG PET-CT metabolic index for nasopharyngeal carcinoma. J Cancer Res Clin Oncol 136: 883-889, 2010

26. Kubota R, Yamada S, Kubota K, Ishiwata K, Tamahashi N and Ido T: Intratumoral distribution of fluorine-18-fluorodeoxyglucose in vivo: High accumulation in macrophages and granulation tissues studied by microautoradiography. J Nucl Med 33: 1972-1980, 1992. 